\title{
Information Technology and problem solving in mathematics education
}

\author{
G. Dettori, S, Greco, E. Lemut \\ Instituto Matemetica Applicata, C.N.R. \\ Via de Marini 6 \\ 16149 Genova, Italy \\ E-mail:Dettori@ima.ge.cnr.it
}

$1 \quad$ WHAT IS IT?

Several slightly different definitions of IT can be found in the technical literature: basically all of them state that IT:

- is the confluence of informatics with other technologies;

- is aimed at the processing and communication of information;

- has many social, economic and individual implications.

The rapid development of IT and the permeation of its use in almost any field of human activity have brought about profound cultural and social changes which require corresponding changes in educational systems.

\section{INTEGRATION OF IT WITH TOPIC EDUCATION}

IT influences education in several ways: it influences knowledge and the need for knowledge. When technology was first introduced into education, the focus was mainly on technology itself; since then, the focus has been more and more shifted from technology to the knowledge to be taught and learned; and technology is viewed more and more as a means to mediate learning. This does not mean that IT should not be studied also as a discipline by itself; in fact, basic competencies should be taught to students independently of other disciplines - as general knowledge that can be useful outside school or as a tool for learning.

Capacity Building for IT in Education in Developing Countries

G. Marshall \& M. Ruohonen (Eds.)

ㅅ) 1998 IFIP. Published by Chapman \& Hall 
When microcomputers became widespread in schools, preoccupation with computer literacy began and mathematics courses started including a self-standing informatics module, aiming to become familiar with the computer. Over the years curricula have been modified more and more in order to integrate IT into teaching school subjects. This implies that computer literacy is becoming less a subject in itself and more a practical way to understand potentialities and limits of computers, finalised to its application. Students gain experience with computer systems while using them and, at the same time, learn to appreciate them as a tool which helps in solving problems, in fomalising ideas and in investigating.

Problem solving has been a field of study in cognitive psychology for years, as concerns general learning, not just in conjunction with mathematics or other scientific disciplines. At present, the name 'problem solving' indicates an active processing of information, which involves more than a set of general strategies; problem-solving ability is domain-dependent and cannot be easily generalised to a wide range of domains.

When teachers want to emphasise problem solving in a curriculum, they must play the role of mediators of learning rather than transmitters of knowledge. This entails adopting a cognitive view of learning in which students construct meanings through their experiences, rather than barely teaching algorithms and finding learning environments that facilitate experiential learning and support problem solving activities.

Concerning mathematics, problem solving does not mean simply to assign students some problem, asking them to find a numerical solution. It is an approach to learning where theoretical concepts are introduced during the solution of a problem. This is useful from three points of view:

- it motivates the introduction of theoretical points, showing that they have an actual applicability;

- it eases the introduction of new topics, since they are learned 'by examples' and 'by doing' hence making them more concrete;

- it makes teachers and students aware of preconceptions, which sometimes hinder learning (cultural obstacles).

Moreover, to make problem solving even more motivating and stimulating, particular attention should be paid to the choice of problems that should be related to some problematic situation somehow familiar in the students' context.

For all these reasons, problem solving should be proposed, including not only standard problems containing a complete problem description and all data, but also problems without numerical data and open or semi-structured ones, from which students must develop mathematics. These situations help them open up their minds and look deeper into problems and offer occasions to connect mathematics education with other disciplines, in particular with developing good expressive capabilities. 
Hence problem solving is an application-based approach to mathematics and this has been found a necessary condition for a mathematics education program to be emancipatory and empowering, tuning students' learning with society's needs. On the other hand, a formal and theoretical mathematics program is often used as a way to discriminate and marginalise some sectors of society.

WHY IT AND PROBLEM SOLVING?

Several reasons motivate the application of IT in education at all levels of mathematics learning:

- the need to support the processes of abstraction;

- the need to mediate learning by means of representations;

- the need to relieve students of work-intensive technical operations (such as graphing or heavy number computation.

As concerns representation in particular, the research in mathematics education has highlighted its importance both in problem solving and to mediate the introduction of abstract concepts. It has also been pointed out that using in parallel different representations for the same topic can be even more fruitful if they are correctly and strongly linked, since this allows one to emphasise different aspects or properties of the concepts under consideration hence leading to a deeper understanding and to a better capability to apply the learned concepts to new situations. Several representations of a concept can co-exist in somebody's mind, complement each other and eventually get integrated into a single, more general representation of that concept. This process of integration is the base of abstraction (that is, the ability to shift attention from instances of objects to their structure, properties and relationships), which is an essential component of mathematical thinking and, in general, of mature cognitive capabilities. From this point of view, the computer appears an excellent tool since it represents a fundamental advance in our representational capabilities. In fact, it makes it possible to represent mathematics with an amount of structure not offered by any other tool.

Moreover, using IT to promote activity-based learning brings about a change in students' learning style, making them more involved and responsible for their mathematics education. This also brings about a change in students' beliefs about what doing mathematics means. Hence this use of IT and problem solving in math education favours the transformation from a passive leaning style based on copying and memorising to a learning style based on action and direct experience.

\section{6}

\section{POTENTIALITIES FOR IT IN EDUCATION}

Lab activities in mathematics education can exploit, either for introducing and for deepening concepts, the characteristics of software of different kinds, such as educational software, commercial software or programming languages.

Computers are very particular tools with characteristics different from any other used in school. Their potentialities stem from their capabilities of interaction; their limits from the fact that their way of interacting can follow only fixed schemes, 
depending on the running program, without any personal possibility of changing the thread of discussion.

Computers allow fast computations, visualisations of two- and three-dimensional objects, simulation of casual events with several advantages:

- to discuss a mathematical concept under different points of view, which usually leads to a deeper understanding and a better mastery of concepts (for instance, geometrical transformations can be completely described by means of equations but using a graphical representation clarifies better the concept);

- to change the presentation order of some concepts, based on their relative degree of difficulty (for instance, the resolution of equations can be introduced graphically before completing the study of algebraic manipulation rules);

- to focus on resolution strategies rather than on performing calculations which can improve problem-solving activities;

- to emphasise the meaning of math concepts before being able to formally handle them (for instance, derivatives computed by some software can be used to find minima and maxima of a function based on their meaning, before learning to compute them).

This approach implies a redefinition of a teacher's role and a revision of curricula in order to give more space to non-routine activities apt to stimulate the student's creativity. Moreover, learners usually have different background and experience, and this influences the impact of IT on their learning.

The first attempts to introduce IT in education were made at the beginning of the 1960 s with systems whose design and aim was strongly influenced by the hardware and software at the disposal at that time (only mainframes, usable in timesharing; few programming languages for the construction of systems; little application software). A change of approach and use over the past 35 years is due also to the availability of new hardware and software tools which are cheaper, more ductile user friendly, and with a range of features.

Technological development has produced a growing distance between the basic functioning of computers and the way computers interact with users. It has produced new, more intellectual and virtual ways to use them.

This evolution has been influenced by the research in education; it has gone in parallel with a different conception of teaching and learning, underlined by changes in the official school programmes.

\section{8}

AN OVERVIEW OF APPROACHES TO USING IT IN EDUCATION

It is not our purpose to give a history of IT in education. However, summarising general trends can help us understand the present and prepare for the future.

A precise classification of software tools which have been used in education over the years is rather difficult since often one kind of tool evolved into another, so that several tools belong to more than one group we are going to mention. 
Systems have evolved under several respects: focus, structure and interface. As concerns focus, it has shifted from seeing the computer as a tutor to using it as a tool to giving a central roles to students' activity. As concerns structure, software has evolved from rigidly organised systems to more and more flexible ones. As concerns interfaces, they are becoming more and more articulated and user-friendly, supporting communication in different forms, as is the case of multimedia systems, where the concept of interactivity acquires a new dimension by focusing on individual strategies during the learning process and encouraging the exchange of knowledge in various forms between the system and the learner.

\subsection{CAI/CAL system}

The attempt to use technological tools in education began in the early 1960s with the design and implementation of computer-assisted instruction/computer assisted learning systems, which offered individualised pacing, provided reinforcement and evaluated performance. They were generally limited to present some topics. The didactic path was very rigid, based on a 'stimulus-response' model, with a oneway teaching interaction. A system posed some questions and/or problems and evaluated the student's answers based on a rigid predefined schema. Hence the behaviour of the system was not adaptable to the different needs of students. The merit of $\mathrm{CAI} / \mathrm{CAL}$ is that they have been a first attempt to use technology to mediate learning in a period when the use of computers was mainly based on programming with languages which required one to adapt one's own thinking to the way computers worked.

\subsection{Intelligent tutoring systems ICAI/ILE}

The need to get over the limits of CAI encouraged studies in cognitive psychology and artificial intelligence, with particular attention to the interaction between teacher and student. These researches led to the development of new educational systems called ICAI (Intelligent Computer-Assisted Instruction) or Intelligent Tutoring Systems (ITS). In an ITS a student can exploit a two-way dialogue, somehow personalised to his/her needs. Some ITSs give students a well-defined didactic path; others let students free to choose their own paths; other mix the two approaches. An ITS is a collection of expert systems (that is, systems planned to emulate the behaviour of human experts on some determined topic), suitably designed to interact with each other, such as (at least):

- an expert module, experienced on the topic to be taught (this module is needed to analyse, understand and solve problems on that topic either proposed by the student or by the system itself);

- a tutoring module, experienced in some didactical methodology, able to handle problems related to the learning process;

- a student module, that is, a knowledge base concerning the learning status of the students as gathered by the system through a dialogue (a qualitative model, dynamically improved and increased, which allows the system to personalise the teaching dialogue, and, in some way, to direct the learning path); 
- $\quad$ an interaction module to make the system easy and pleasant to use, and to allow both the system and the student to take the initiative in the dialogue.

ITSs have been objects of research for many years and have evolved towards more and more flexible configurations in order to adapt them to the behaviours of the users. This has gradually led to design software structured more as learning environments where the user has freedom to explore the topic under consideration and to pay more attention to the design of interfaces based not only on text but on different media, used not simply to make the system more appealing, but actually as dialogue tools. These changes opened the way to the creation of microworlds and multimedia.

\subsection{Microworlds - learning through exploration with constraints}

The concept of microworld was first formulated in 1971 by Marvin Minsky and Seymour Papert within the Artificial Intelligence community in order to communicate the idea of problem solving in a simple and constrained domain. Since then the goal for microworlds has shifted - from teaching computers to solving problems to designing learning environment for the acquisition of knowledge, making personal discovery a part of mathematical education. Microworlds explicitly intend to develop an investigative attitude towards mathematical enquiry.

Their basic features include:

- providing the user with a visual language which allows the generation and manipulation of objects in order to produce visual representation of a problem's situation or solution step;

- offering several different ways to achieve a goal;

- allowing a direct manipulation of objects;

- embodying a model of an abstract knowledge domain of visual, manipulable representation mediates between the concrete and directly manipulable on one side and formal and abstract on the other side.

A free exploration of a microworld (or other system) offers learners a rich experience but does not guarantee that specific learning occurs. Hence the system requires a suitable using environment organised by the teacher.

The best-known and most widely used examples of microworlds are the Logo programming environment and the graphic system Cabri-geometre. Cabri-geometre is a direct manipulation system designed and implemented for educational purposes, in particular for the study of Euclidean geometry, Euclidean geometry represents a synthetic view of geometry and is usually more difficult than analytic geometry. It is still controversial which of the two approached should be presented first at school. Cabri gives a contribution to smooth and make appealing this approach to geometry, The system is available for both Macintosh and PC computers and is used in many schools of different kinds at different levels. It offers pure drawing primitives - for lines, points, polygons, geometric primitives to build geometric objects with some given properties and in relation to other objects already drawn, and it allows a high degree of interaction by moving the constructed 
objects yet preserving the original properties and relations. It has a user-friendly interface and is easy to learn. A problem with its use is that it does not have the possibility to choose or produce random elements.

\subsection{Hypertexts, hypermedia and multimedia}

These terms refer mainly to the structure and operating mode of systems.

Hypertexts breaks the traditional sequentiality of texts, subdividing materials into modules variously connected with each other to form a graph structure so that users can create their own personalised reading path.

The term 'multimedia' refers to systems that combine information in different formats (texts, images, sounds, video and animation) to communicate more effectively. Multimedia achieve a high degree of interactivity between user and the system since non-textual input/output is not used simply to make the interface more pleasant but is strictly part of the handled knowledge.

Hypermedia systems combine the non-linear structure of hypertext and the variety of interaction forms of multimedia, giving rise to a new concept of interactivity where the user is a fundamental component determining the starting point, the aim and the form of the interaction.

As concerns content, multimedia systems often, but not necessarily, include some microworld with excellent results since these two approaches are both learnerand activity-centred.

\subsection{Perspectives - cooperative learning by means of Internet}

The current trend is to add communication technologies to IT and to realise systems that allow a meaningful and complex exchange of information, ideas, solutions and materials, by using the facilities of Internet. This long distance, realtime, structured communication will probably be a good way to overcome the isolation of off-centred locations, allowing students to share learning outside their school environments. This approach can increase motivation, foster group work and discussion, stimulate writing and communication skills, and open up minds with the acquaintance of different social and cultural contexts.

Other kinds of software, mainly developed for commercial purposes, have undergone a parallel process of evolution and have been successfully applied in mathematics teaching.

\subsection{Software packages - professional and educational}

\section{Spreadsheets and other tools}

Software packages, both professional and educational, are often successfully used in school, in particular spreadsheets, symbolic manipulators, graphics systems, statistics packages, and ad hoc systems with mixed capabilities. From an educational point of view, the increased use of general purpose commercial software has put an emphasis on the information handling aspects of students' work. Their use requires from the teacher a working out of educational approaches in order to avoid using them to find numerical results rather than as learning tools. 
Spreadsheets have been created as professional tools. They are table builders with a labelling systems for rows and columns and facilities to draw graphs based on the constructed tables. Their most salient feature is the facility to express formulas making reference to cells' addresses and using them to fill a table based on input or previously computed values. Other facilities are the possibility to insert, delete and move columns or sets of cells, hence adding a dynamic aspect to the produced tables. It is clear that they are a generic tool as concerns mathematics education, which makes them suitable for numerous topics - from a first approach to algebra to the introduction of function, to statistical analysis of data, etc. Due to their nature, problem solutions based on them are essentially numerical computations based on a trial-and-error strategy, rather than formal solutions based on some mathematical theory. The two best-known, Lotus and Excel, differ only in a few features in the interface, and are essentially equivalent as concerns their use.

Computer algebra systems are mostly general-purpose mathematical manipulation systems. offering the possibility to perform symbolic manipulation, calculus, number theory, linear algebra, statistics and differential equations. In high school teaching usually only symbolic manipulators (such as Derive or Theorist) with a restricted range of operations and graphing capabilities are applied since they are easier to learn and do not require big memory occupations. Richer systems, which require more memory space and offer programming possibilities, Maple or Mathematica, for example, are used more for professional purposes or at the university level. Despite their use in school in several countries, computer algebra systems as cognitive technology for mathematics are considered to be still a field with great potential for evolution.

\section{Graphical and geometric software}

Among graphical programs that have been successfully used in school for supporting geometry learning the most widely-known are Cabri-geometre, Geometer Sketchpad or the graphical part of Logo. All these systems differ from drawing tools (MacPaint, for example) where the process of construction of a drawing involves only action, in that they require an explicit description of figures in terms of their geometric properties, either by selecting a menu item or by writing a list of commands. This is valuable from an educational point of view since the description of a drawing is strongly related to the deep structure of geometry. In them a construction task aims not simply to produce a drawing but to produce a description resulting in a drawing. Moreover they offer facilities to modify a drawing yet preserving the intended properties.

\section{Proramming}

Computer programming has been present in education for many years. The initial absence of appropriate high-level programming environments resulted in the widespread use of teaching students some very limited programming. At present, though languages are usually embedded in better operating environments which may stimulate their educational use, the use of programming in math courses is not as popular as it was years ago. One of the reasons is that attention is now more on what computers can do to help us solve problems than on the inner-working of computers themselves. 
Programmable environments have been often regarded as superior to ready-made software packages since students can learn that they can construct and modify working tools. However programming is a difficult discipline that cannot be learned in a few weeks. It is impossible, in the limited time that a mathematics teacher can dedicate to teaching programming, that students can gain such a mastery to be able to develop anything more than simple exercises, which makes programming a suitable topic only if complemented with other environments.

\section{9}

\section{TOPICS FOR DISCUSSION}

How much awareness of the historical evolution of technology should students and teachers acquire? Is it necessary that developing countries go through all steps developed countries have gone through?

Compare national situations:

- How diffused are computers in schools? What kinds of hardware and software are available?

- How extensively are computers used? For what kinds of activities?

- What is planned by official programs?

- How are teachers trained and supported to carry out activities?

- Is teaching based on a problem solving approach? 